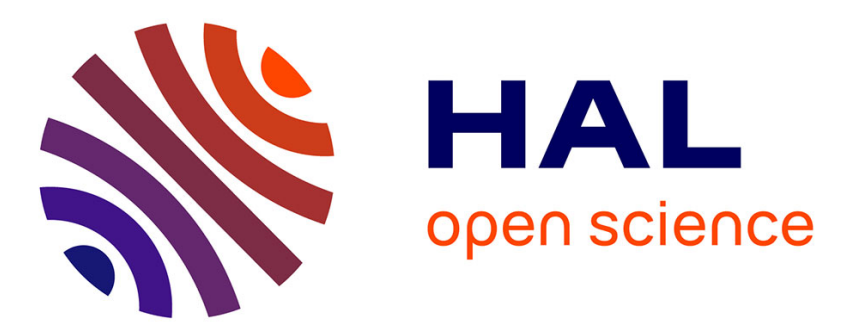

\title{
Rayleigh-Bénard experiment in liquid helium ; frequency locking and the onset of turbulence
}

\author{
J. Maurer, A. Libchaber
}

\section{To cite this version:}

J. Maurer, A. Libchaber. Rayleigh-Bénard experiment in liquid helium; frequency locking and the onset of turbulence. Journal de Physique Lettres, 1979, 40 (16), pp.419-423. 10.1051/jphyslet:019790040016041900 . jpa-00231657

\section{HAL Id: jpa-00231657 https://hal.science/jpa-00231657}

Submitted on 1 Jan 1979

HAL is a multi-disciplinary open access archive for the deposit and dissemination of scientific research documents, whether they are published or not. The documents may come from teaching and research institutions in France or abroad, or from public or private research centers.
L'archive ouverte pluridisciplinaire HAL, est destinée au dépôt et à la diffusion de documents scientifiques de niveau recherche, publiés ou non, émanant des établissements d'enseignement et de recherche français ou étrangers, des laboratoires publics ou privés. 


\title{
Rayleigh-Bénard experiment in liquid helium ; frequency locking and the onset of turbulence
}

\author{
J. Maurer and A. Libchaber \\ Groupe de Physique des Solides de l'Ecole Normale Supérieure, 24, rue Lhomond, 75231 Paris Cedex 05, France
}

(Reçu le 7 mai 1979, révisé le 21 juin 1979, accepté le 29 juin 1979)

\begin{abstract}
Résumé. - Sur une expérience de Rayleigh-Bénard dans l'hélium liquide nous étudions les régimes dépendant du temps, au-dessus du seuil de convection. La cellule expérimentale est de géométrie rectangulaire présentant deux rouleaux de convection de section aplatie, $\alpha=1,90, \alpha$ nombre d'onde des rouleaux. Une première fréquence $f_{1}$ d'oscillation des rouleaux apparaît pour un nombre de Rayleigh $R \sim 2 \times 10^{4}$, puis une deuxième fréquence $f_{2}$, beaucoup plus basse, pour $R \sim 2,7 \times 10^{4}$. Deux accrochages de fréquences sont observés, avec hystérésis, pour les rapports de fréquence $f_{1} / f_{2}=6,5$ et $f_{1} / f_{2}=7$. De ce dernier accrochage germine le mouvement turbulent des rouleaux pour $R \sim 3,4 \times 10^{4}$. Cette transition vers la turbulence apparaît par génération des fréquences $f_{2} / 2$, $f_{2} / 4, \ldots$ et en temps réel par un chaos de phase plutôt que d'amplitude.
\end{abstract}

\begin{abstract}
In a Rayleigh-Bénard experiment in liquid helium, we study the time-dependent regimes, above the onset of convection, in a parallelepipedic cell with two convective rolls of oblate cross-section $\alpha=1.90$, where $\alpha$ is the rolls wave number.

A first frequency $f_{1}$, associated to oscillating rolls, appears for a Rayleigh number $R \sim 2 \times 10^{4}$, then for $R \sim 2.7 \times 10^{4}$ a second frequency $f_{2}$, much smaller, is observed. Two frequency locking regimes are present, with hysteresis, for the frequency ratios $f_{1} / f_{2}=6.5$ and $f_{1} / f_{2}=7$. The onset of turbulence germinates from the last frequency locking state for $R \sim 3.4 \times 10^{4}$. This transition to turbulence is triggered by the generation of the frequencies $f_{2} / 2, f_{2} / 4, \ldots$ and in real time is defined by a phase-chaotic behaviour rather than by an amplitude behaviour.
\end{abstract}

We present in this Letter some new results on a Rayleigh-Bénard experiment whereby a parallelepipedic cell of liquid helium is heated from below, the liquid being at a temperature of $3.00 \mathrm{~K}$ under a pressure of 3 atmosphere, its Prandtl number being $\sigma=v / \kappa=0.5$. In such an experiment, as one increases the temperature difference $\Delta T$ between the top and bottom part of the fluid, the first bifurcation is associated with fluid convection. In our geometry, a parallelepipedic cell with a base $1.6 \times 2.8 \mathrm{~mm}$ and a height $0.85 \mathrm{~mm}$, we have studied the metastable state where two convective rolls, perpendicular to the largest side of the sample, are formed. Figure 1 shows the curve of marginal stability for the onset of convection. Whereas for a sample of infinite transverse dimension the roll wave number [1] is equal to $\alpha_{c}=3.117$, our metastable state corresponds to $\alpha=1.90$ ( $\alpha$ is a normalized wavevector, $\alpha=2 \Pi d / \lambda$ where $d$ is the cell height). The second bifurcation, for a small Prandtl number fluid, leads to a limit cycle, physically associated to waves propagating along the convective rolls, the so-called oscillatory [1] instability. We detect, with a local bolometer, a temperature oscillation of frequency $f_{1} \simeq 550 \mathrm{mHz}$ at the onset for a Rayleigh number $R \simeq 2 \times 10^{4}$, or a temperature difference $\Delta T \simeq 120 \mathrm{mK}$. The third bifurcation generates a second frequency, $f_{2}$, the onset of which is for $R=2.7 \times 10^{4}$ and its starting value $f_{2}=90 \mathrm{mHz}$ Then, as one keeps increasing the temperature difference, all the combination frequencies, $f=m f_{1}+n f_{2}$, $m$ and $n$ integer, appear as one Fourier analyses the data (Fig. 2). This is followed by two frequencylocking states (Fig. 3), for Rayleigh numbers between $3 \times 10^{4}$ and $3.3 \times 10^{4}$, the first one corresponds to $f_{1} / f_{2}=6.5$, and the second one to $f_{1} / f_{2}=7$ (Fig. 4). Finally, for $R=3.38 \times 10^{4}$, the onset of turbulence is observed, germinating from the last locking state, with a generation of the frequencies $f_{2} / 2, f_{2} / 4$ and so on (Fig. 6).

In the following we will briefly describe the experimental situation and then develop a number of important results of the experiment. 


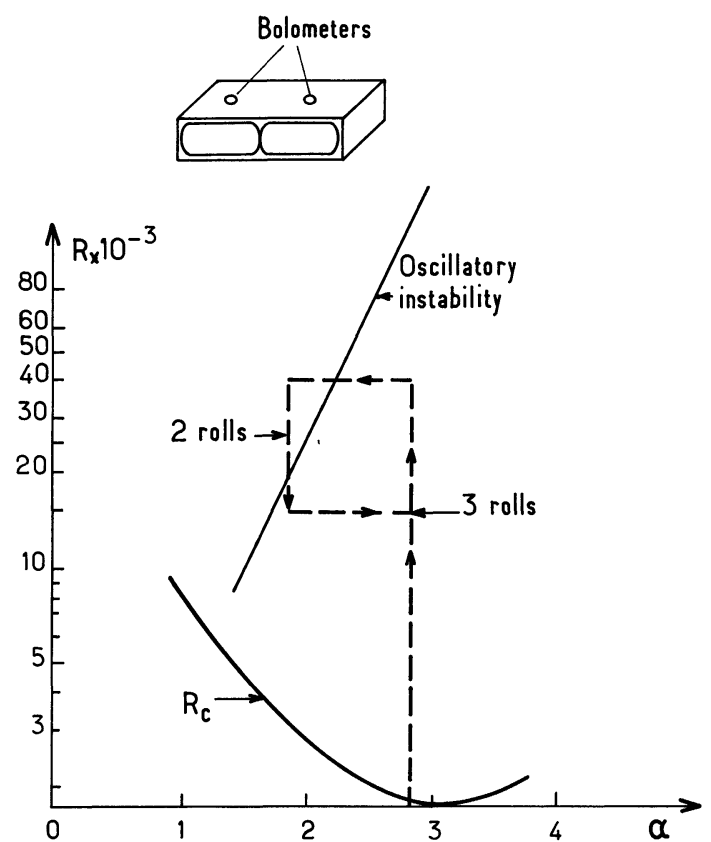

Fig. 1. - Marginal stability curve for the onset of convection $\left(R_{\mathrm{c}}\right)$, and the onset of oscillatory instability as a function of the roll wave number [1]. The dashed lines present the experimental procedure to obtain a sample with two convective rolls. The insert is a schematic of the experimental cell with the bolometers.

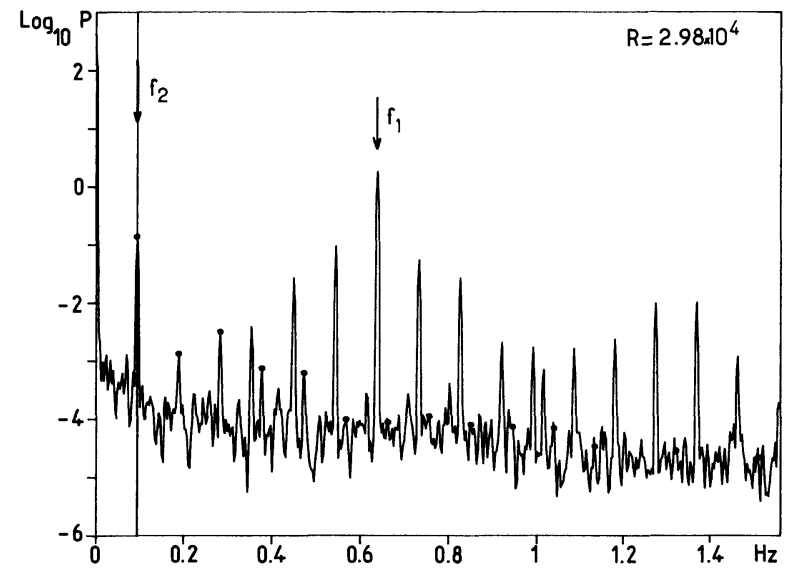

Fig. 2. - Typical Fourier spectrum above the third bifurcation. $R$ is the Rayleigh number. The two frequencies $f_{1}$ and $f_{2}$ are not commensurate. The black dots represent the harmonics of $f_{2}$. All the lines can be interpreted as combinations of $f_{1}$ and $f_{2}$ (bandwidth $0-1.5625 \mathrm{~Hz}$. Averaging time $600 \mathrm{~s}$ ). Vertical units are arbitrary.

1. Experimental situation. - The overall set-up is similar to the one described in our previous letter [2], the main differences being the cell geometry and the presence of two local bolometers instead of one.

The temperature of the experimental cell is $3.00 \mathrm{~K}$, with a temperature regulation of $10^{-6}$. The applied pressure, 3 atmospheres, is not regulated, but the cell is in contact with a large ballast volume of constant temperature, so that the pressure stability is better than $10^{-2}$ atmosphere.

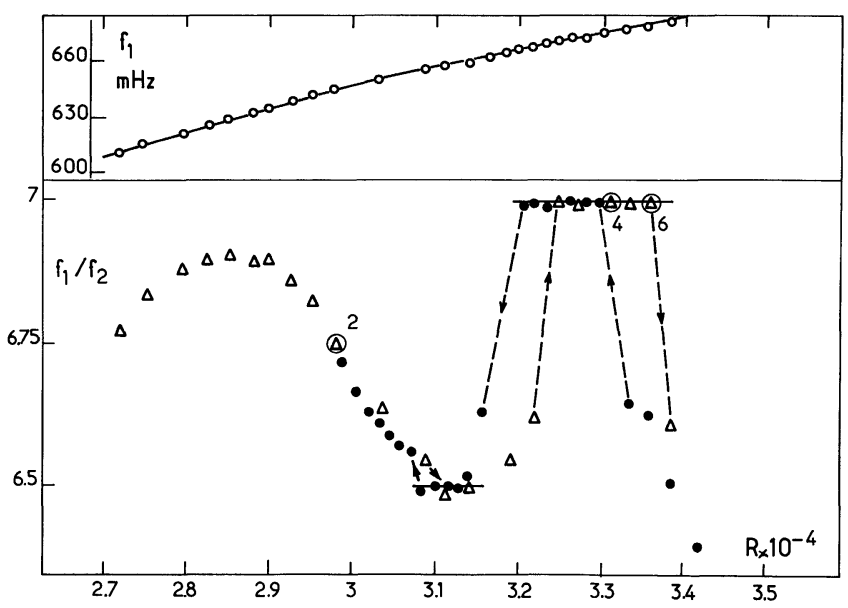

Fig. 3. - Ratio $f_{1} / f_{2}$ as a function of the Rayleigh number. Also, top curve, evolution of $f_{1}$. The triangles are for increasing $\Delta T$, the black points for decreasing $\Delta T$. The points $2,4,6$ correspond to figures $2,4,6$.

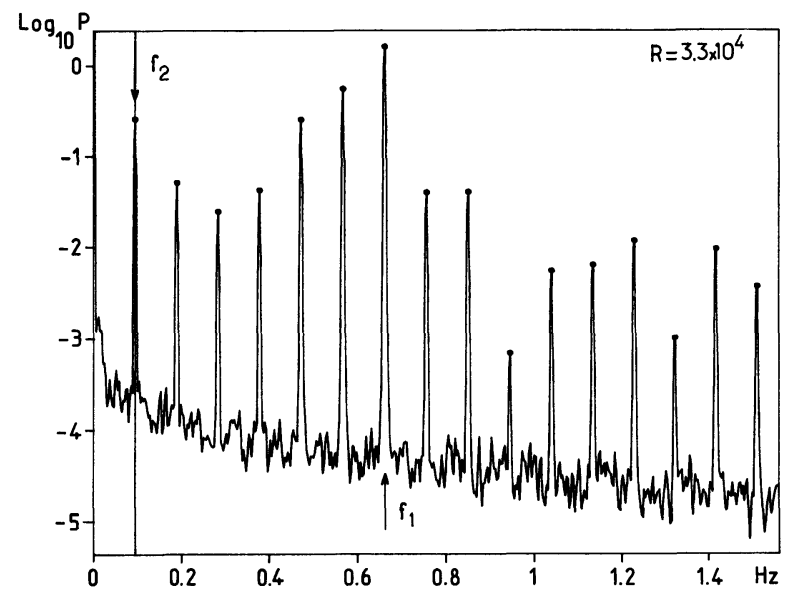

Fig. 4. - Fourier spectrum corresponding to the frequency locking regime $f_{1} / f_{2}=7$. The black points correspond to harmonics of $f_{2}$ (bandwidth 0-1.562 $5 \mathrm{~Hz}$. Averaging time $1.35 \times 10^{3} \mathrm{~s}$ ). Vertical units are arbitrary.

The cell geometry is essentially similar to the one used by Gollub and Benson [3], with all dimensions scaled down by a factor of 10 . This is done in order to obtain two rolls of small wave number, as shown by Gollub's optical observation. The cell is of rectangular shape, with a base $1.6 \times 2.8 \mathrm{~mm}$ and a height $0.85 \mathrm{~mm}$. It is machined out of araldite with a precision of about 10 microns. The cell dimensions are thus the most unprecise parameters of the experiment.

The upper part of the cell is made out of a sapphire crystal within which two small bolometers are located, as described in reference [2]. (See also the insert of figure 1.)

The bolometers consist of two small carbon discs of diameter $0.4 \mathrm{~mm}$ and thickness $0.2 \mathrm{~mm}$, obtained from an Allen-Bradley resistor. The two bolometers 
Table I.

$\begin{array}{cccccccccc} & \mathrm{mm} & \mathrm{mm} & \text { deg. } & \text { atm. } & \text { poise } & \mathrm{cm}^{2} / \mathrm{s} & \mathrm{cm}^{2} / \mathrm{s} & \sigma & \mathrm{s} \\ \text { cell } & \text { base } & d & \text { temp. } & P & 10^{6} \eta & 10^{4} \kappa & 10^{4} v & & d^{2} / \kappa \\ - & - & - & - & - & - & - & - & - & - \\ & 2.8 \times 1.6 & 0.85 & 3.00 & 3 & 43 & 5.75 & 2.9 & 0.5 & 12.5\end{array}$

are positioned so that they are each at the centre of a convective roll, as sketched in figure 1 . Their sensitivity, at $3 \mathrm{~K}$, is about $\frac{\Delta R}{R} \simeq \frac{4 \Delta T}{T}, \Delta R$ represents the change of resistance of the bolometer when the temperature is displaced by a value $\Delta T$.

The lower part of the cell is made out of copper. The parameters of the experiment, fluid and dimensions, are given in table I.

2. Metastability of the convective rolls. - It is difficult in helium (no visual observation) to determine the shape and orientation of the convective rolls. So we have to rely on the very systematic study of Stork and Muller [4], and the optical observation of Gollub [3] and Berge [5], where the general agreement is that rolls tend to align perpendicular to the largest side dimension.

It is also important in this experiment to describe precisely the past history of the fluid convective state. In order to reach a state with two rolls in the sample, perpendicular to the largest side dimension as in figure 1, the experimental procedure is as follows. We increase the temperature difference $\Delta T$ from zero, and measure the thermal conductivity through the sample. We observe the transition to convection for a Rayleigh number $R=2300$. This transition is associated with the presence of three rolls in our cell, clearly observed in analysing the DC temperature of our two top plate bolometers. In a three-rolls pattern, one of the bolometers is at a higher temperature than the other as it experiences the arrival of hot fluid, while the second one is at the location where cold fluid is going down. Also three rolls are consistent with a cell $0.85 \mathrm{~mm}$ in height and $2.8 \mathrm{~mm}$ for the largest side dimension.

If we keep increasing the temperature difference, at a Rayleigh number of the order of $R \simeq 4 \times 10^{4}$, a transition to a two-rolls pattern [6] is observed, the two bolometers jumping to the same local temperature as they should with a two-rolls pattern (Fig. 1). The rolls wave number is then

$$
\alpha=2 \Pi \times \frac{0.85}{2.80} \quad \alpha=1.90 .
$$

This metastable state, with two rolls in the sample, can then be kept, as we lower $\Delta T$, down to a Rayleigh number of $R=1.5 \times 10^{4}$ where it jumps back to three rolls. We thus trace an hysteresis cycle for the effective thermal conductivity. All our data, presented here, will be with two rolls present in the sample.
3. Importance of the roll wave number $\alpha$ for the onset of convection and of time-dependent oscillations. Going back to figure 1, one sees that the wave number $\alpha$ affects the Rayleigh number for the onset of convection, and also the Rayleigh number for the onset of the oscillatory instability. For an infinite sample with rigid boundary conditions but free lateral boundaries, convection starts [7] for $\alpha_{\mathrm{c}}=3.117$ and $R_{\mathrm{c}}=1707$. Our state with two rolls, corresponding to $\alpha=1.90$, should have a higher Rayleigh number as predicted by the curve of marginal stability on figure 1. But, as described in the last paragraph, we cannot measure this number in the experiment.

This is the reason why all our data are given in Rayleigh numbers $R$ instead of $R / R_{\mathrm{c}}$. Given such a low wave number, the onset of the oscillatory instability should occur for a low Rayleigh number, as predicted by Busse's theory, and this is the case [8], $R=2.09 \times 10^{4}$. Evidently Busse's theory is only valid for an infinite sample. In our case, a sample of finite size, with a roll length of the same order as the wavelength of the oscillatory instability this theory is only a guide and at best qualitative. Figure 3 is a typical recording above the third bifurcation, of the two frequencies $f_{1} f_{2}$ and their combination. On the $Y$ axis LOG MAG DB indicates the logarithm of the signal amplitude in decibels.

4. Frequency locking regime. - In this experiment more than two main frequencies are never measured until we reach the turbulent regime, in confirmation with other observations $[2,3,9]$.

One of the new results of this paper is the observation of frequency locking in our two-frequency operation. As shown on figure 3 we observe frequency locking for $f_{1} / f_{2}=6.5$ and $f_{1} / f_{2}=7$. The Fourier analysis data are shown on figure 4 for $f_{1} / f_{2}=7$.

Frequency locking in non linear systems with two frequency operations have been observed in various physical systems by Rayleigh, Van der Pol and W. E. Lamb Jr. [10]. They occur in classical sustained oscillators where the locking mechanisms compete against the sustaining force. In our case, we are in a phase-locking regime where the phase of each frequency is modulated by the other. We will quantitatively analyse our phase-locking phenomena in another publication.

The data shown on figure 3 for $f_{1} / f_{2}=7$ show a strong hysteresis for the locking regime, and a large forbidden gap in the frequency ratio. In real time recording this locking state is extremely stable [11]. 
To close this section, we show on figure 5 another characteristic of this regime, the very sharp line spectra one observes in the frequency locking regime, where $f_{1} / f_{2}=7$. Although we are still limited by our data acquisition system, the quality factor of the $f_{1}$ line is better than 1500 .

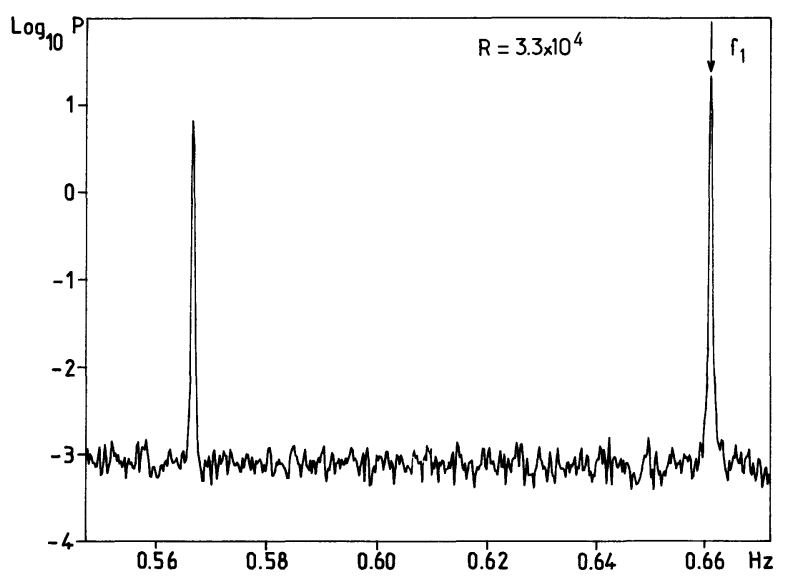

Fig. 5. - Sixth and seventh harmonics of $f_{2}$ in figure 4 (the seventh harmonic corresponds to $f_{1}$ ). This Fourier recording is for a long averaging time, and shows the line shape and linewidth in the locking state $f_{1} / f_{2}=7$. (Bandwidth $547.5-672.5 \mathrm{mHz}$. Averaging time $14 \times 10^{3} \mathrm{~s}$.) Vertical units are arbitrary.

5. Onset of turbulence. - Turbulence seems to germinate from the locking state $f_{1} / f_{2}=7$.

It looks as if locking of the two frequencies is the best chance for the system to keep its order. We show on figure 6 the Fourier spectra for $R=3.38 \times 10^{4}$. One clearly observes sidelines at the frequencies $f_{2} / 2$ and $f_{2} / 4$, i.e. the frequencies $m f_{2}-\frac{f_{2}}{2}$ and $m f_{2}-\frac{f_{2}}{4}$. Turbulence intuitively appears as a softening [12] of the low frequency $f_{2}$, with parametric amplification of submultiple frequencies.

If we analyse our direct recordings before any Fourier transform, we observe that in the frequency locking regime the two frequencies $f_{1}$ and $f_{2}$ are phase locked. Above the temperature difference where $f_{2} / 2$ and $f_{2} / 4$ are generated, phase locking disappears and a chaotic phase regime is observed, whereas the amplitude of the signal remains unchanged.

6. Conclusion. - In a Rayleigh-Bénard experiment, the two control parameters are the temperature difference $\Delta T$, and the sample size, i.e. the number of convective rolls, their length and cross section.

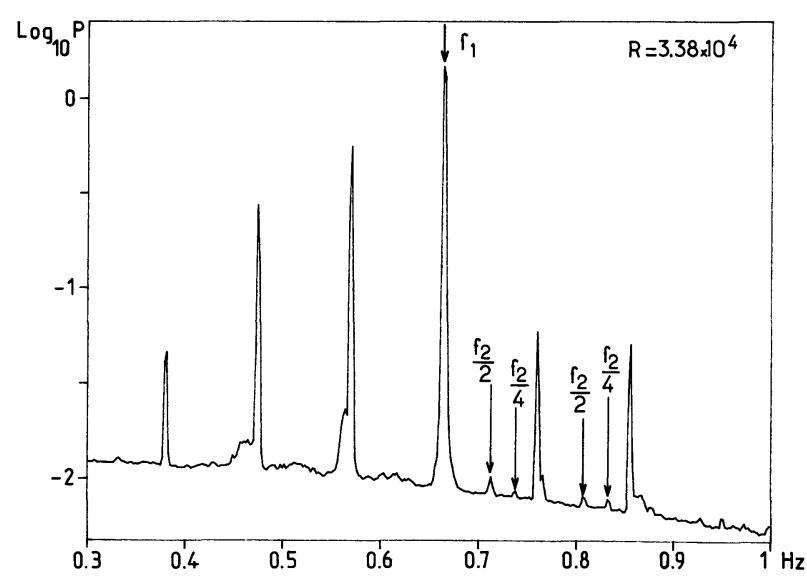

Fig. 6. - Fourier analysis at the onset of turbulence, still in the locking state $f_{1} / f_{2}=7$. The arrows $f_{2} / 2$ and $f_{2} / 4$ correspond to sidelines of the eighth and ninth harmonics of $f_{2}$ (long averaging time). The quantities $f_{2} / 2$ and $f_{2} / 4$ represent the distance from the sidelines to the harmonics. (Bandwidth 0.3-1 Hz. Averaging time $16.5 \times 10^{3} \mathrm{~s}$.) Vertical units are arbitrary.

In our preceding paper [2], using a circular cell, we showed that the various bifurcations were extremely dependent on the aspect ratio $\Gamma(\Gamma=D / 2 d, D$ cell diameter, $d$ cell height). For $\Gamma<3$, a hierarchy of bifurcations was observed, whereas for $3<\Gamma<12$, a very low frequency noise was generated after the onset of convection, for $R \sim 2 R_{\mathrm{c}}$. This confirmed Ahlers [9] observation who even found that for very large $\Gamma$, this low frequency noise threshold almost coincides with the convective one.

The difficulty, with a circular sample, was that we were unable to define the rolls geometry, shape and wave number, which is not the case for a rectangular sample, as described here. We have demonstrated in this paper that two rolls with a small wave number $\alpha<\alpha_{c}$ present frequency locking phenomena, and that the onset of turbulence starts from this locking state with parametric amplification of frequencies $f / 2, f / 4 \ldots$

In conclusion, it seems that the onset of turbulence, and the physical mechanisms leading to it, are extremely dependent on the number of convective rolls and the geometry of their cross section, square or elongated. Evidently, such conclusions are valid only for a small Prandtl number fluid.

Note added in proof. - We have just received a preprint of a paper by Gollub and Benson [13] where they also observe phase locking in a Rayleigh-Bénard experiment in water.

\section{References}

[1] Clever, R. M., Busse, F. H., J. Fluid Mech. 65 (1974) 625. Busse, F. H., J. Fluid Mech. 52 (1972) 97.

[2] Libchaber, A., Maurer, J., J. Physique Lett. 39 (1978) L-369.
[3] Gollub, J. P., Benson, S. V., Phys. Rev. Lett. 41 (1978) 948.

[4] Stork, K., Muller, V., J. Fluid Mech. 54 (1972) 599.

[5] Berge, P., private communication. Dubois, M., Berge, P., J. Fluid Mech. 85 (1978) 641 
[6] A precursor of the three-to-two rolls transition is the observation of local temperature relaxation oscillations of opposite polarity for the two local bolometers. As explained by Busse, as $\Delta T$ increases, the roll wavelength tends to increase and the relaxation oscillations seem to be related to the system, trying to expel one roll in order to increase the roll wavelength, thus to decrease $\alpha$, until it does it at the transition.

[7] Reid, W. H., Harris, D. L., Phys. Fluids 1 (1958) 102.

[8] We have performed various experiments with two rolls of various wave numbers and, whereas for $\alpha=1.90$, $R=2.09 \times 10^{4}$ represents the onset of the oscillatory instability, a Rayleigh number as high as $R=8.5 \times 10^{4}$ is needed for $\alpha=2.7$, in qualitative agreement with figure 1 .
[9] Ahlers, G., Behringer, R. P., Phys. Rev. Lett. 40 (1978) 712.

[10] Lamb, W. E. Jr, Phys. Rev. 134 (1964) 1429.

VAN DER Pol, B., Philos. Mag. 3 (1927) 65.

Lord Rayleigh, Philos. Mag. XIII (1907) 316.

HuYgens, C., 1665. Letters to his father Ouvres complètes, Vol. 5, Société Hollandaise des Sciences, La Haye 1893.

[11] IoOss, G., JoSEPH, D. D., preprint and private communication.

[12] This seems to be connected with one of Ahler's observations.

[13] Gollub, J. P., Benson, S. Y., To appear in Pattern Formation, edited by H. Haken (Springer Verlag). 